\title{
Development of Freeway Weaving Areas Microsimulation Model (FWASIM)
}

\author{
Mahdi Alkubaisi \\ Civil and Infrastructure Engineering Department, Faculty of Engineering and Technology, Al-Zaytoonah University of Jordan, Jordan
}

Received July 22, 2020; Revised August 31, 2020; Accepted September 29, 2020

\section{Cite This Paper in the following Citation Styles}

(a): [1] Mahdi Alkubaisi , "Development of Freeway Weaving Areas Microsimulation Model (FWASIM)," Civil Engineering and Architecture, Vol. 8, No. 5, pp. 1006 - 1018, 2020. DOI: 10.13189/cea.2020.080527.

(b): Mahdi Alkubaisi (2020). Development of Freeway Weaving Areas Microsimulation Model (FWASIM). Civil Engineering and Architecture, 8(5), 1006 - 1018. DOI: 10.13189/cea.2020.080527.

Copyright $\odot 2020$ by authors, all rights reserved. Authors agree that this article remains permanently open access under the terms of the Creative Commons Attribution License 4.0 International License

\begin{abstract}
Comprehensive analysis of traffic behavior requires continuous studies to develop traffic theories explaining that behavior at the microscopic level. The study aims to develop a microsimulation program to evaluate the freeway weaving performance depending on the observed data. FWASIM represents a microscopic analysis of the freeway traffic features. It scans events periodically. The developed FWASIM involves the formulation of driver and vehicle behavior at freeway link, on-ramp, off-ramp, and combine them to produce a flexible, friendly use simulation model. Its concept is mainly depending on the car following and lane change theories. Analytical model validation was conducted based on a comparison of FWASIM output with the VISSIM output. Tests consider the important factors that may affect the traffic behavior for a given segment configuration. The obtained results show agreement between FWASIM and VISSIM outputs. Besides, the field data were used to validate FWASIM. Graphical and t-test methods were used to examine the results. The results are statistically significant which implies that the model provides reasonably accurate measures of effectiveness for the validated range of input data.
\end{abstract}

Keywords Weaving Area, FWASIM, Lane Change, Car Following, Validation

\section{Introduction}

Freeways are considered as the most important elements in the highway system network. Freeway weaving areas represent the critical sections that affect highway capacity and performance. A comprehensive analysis of driver behavior and vehicle attributes within the weaving areas is essential. Such analysis requires continuous studies for development theories to explain the traffic behavior at the microscopic level.

Intelligent Transportation System (ITS) manages and processes the real-time traffic data to be used for the development of online traffic management and operation strategies. To face the increase in traffic congestion, accidents, and transportation delay, a field testing solution is required which is costly and cumbersome. Therefore, microscopic traffic simulation model is considered as a suitable tool to develop vast numbers of online traffic management strategies.

This research paper aims to develop a microsimulation program to evaluate the freeway weaving area performance depending on the observed data. For this reason, the weaving sections of the main freeway named "Mohamed Al-Qasim" street located in Baghdad city were chosen to collect data. Observed data were abstracted, analyzed and then processed to be used as default values throughout the steps of simulation model development.

\section{Research Motivation}

Despite of the availability of variety numbers of microsimulation models, the need for building new or developing the existing programs is still vital task due to the continuous changes in the traffic network. In addition to that reason, the existing microsimulation models are 
operating based on traffic parameters, which are not conforming to that, observed locally, especially the driver parameters. Driver decision play an important role in the position and the speed of each vehicle, which is also influenced by external factors relating to the roadway geometry and traffic interaction. This reason motivates developing simulation models based on the local parameters, observed data, and predicted formulae.

\section{Some Available Microsimulation Models}

Herein below is a brief description of some microsimulation packages, which are used for the analyzing and modeling freeway weaving sections.

VISSIM is a time step based microscopic simulation model developed to analyze the full range of traffic facility operations. The model has been designed for analyzing and modeling transport networks of any size and traffic systems for all functionally classified highways. It can also model a full range of traffic modes such as, passenger cars, buses, light rail, heavy rail, airport facilities, trucks, pedestrians, and bicyclists. The model was developed at the University of Karlsruhe, Germany during the early 1970's [7].

INTEGRATION model was used as an integrated simulation and traffic assignment model during the mid 1980's. The model's approach utilized the same logic for traffic flow to represent both freeway and signalized links. The model represents the traffic flow as a series of individual vehicles follow the macroscopic traffic flow. For this reason, the model is being considered mesoscopic [10].

TRANSIMS is a mesoscopic simulation model, which consists of supporting models, and databases that use advanced techniques to be able analyzing and simulating the integrated transportation system environment. It contributes to the solution of the complexity inherent in transportation issues. Such contributions are that the simulation results addressing traffic congestion, air pollution, energy consumption, traffic safety, and so forth [9].

CORSIM is a microscopic simulation model that tracks the position and movement of each vehicle in the network once each second designed for the analysis and modeling of freeways, networks and basic transit operations. The Federal Highway Administration (FHWA) has developed CORSIM in the mid 1970's. It is a windows-based interface software as it runs within a software environment called the Traffic Software Integrated System (TSIS). The model output can be viewed graphically and assess its performance using animation [12].

\section{Model Formulation}

The model was developed to include the formulation of driver and vehicle behavior at freeway link, on-ramp (merge region), off-ramp (diverge region), forming the highway weaving area, and finally, their combination to produce a flexible and friendly use simulation model. Figure (1) explains the flow chart, which represents the simulation of vehicle behavior at on ramp and the adopted logic to be merged into the freeway link. Flow chart also reflects the main structure of the simulation model. While Figure (2) explains the flow chart for the complete process of simulating vehicles in the freeway and their behavior when attempting to exit from off ramp or from the freeway segment [3]. 


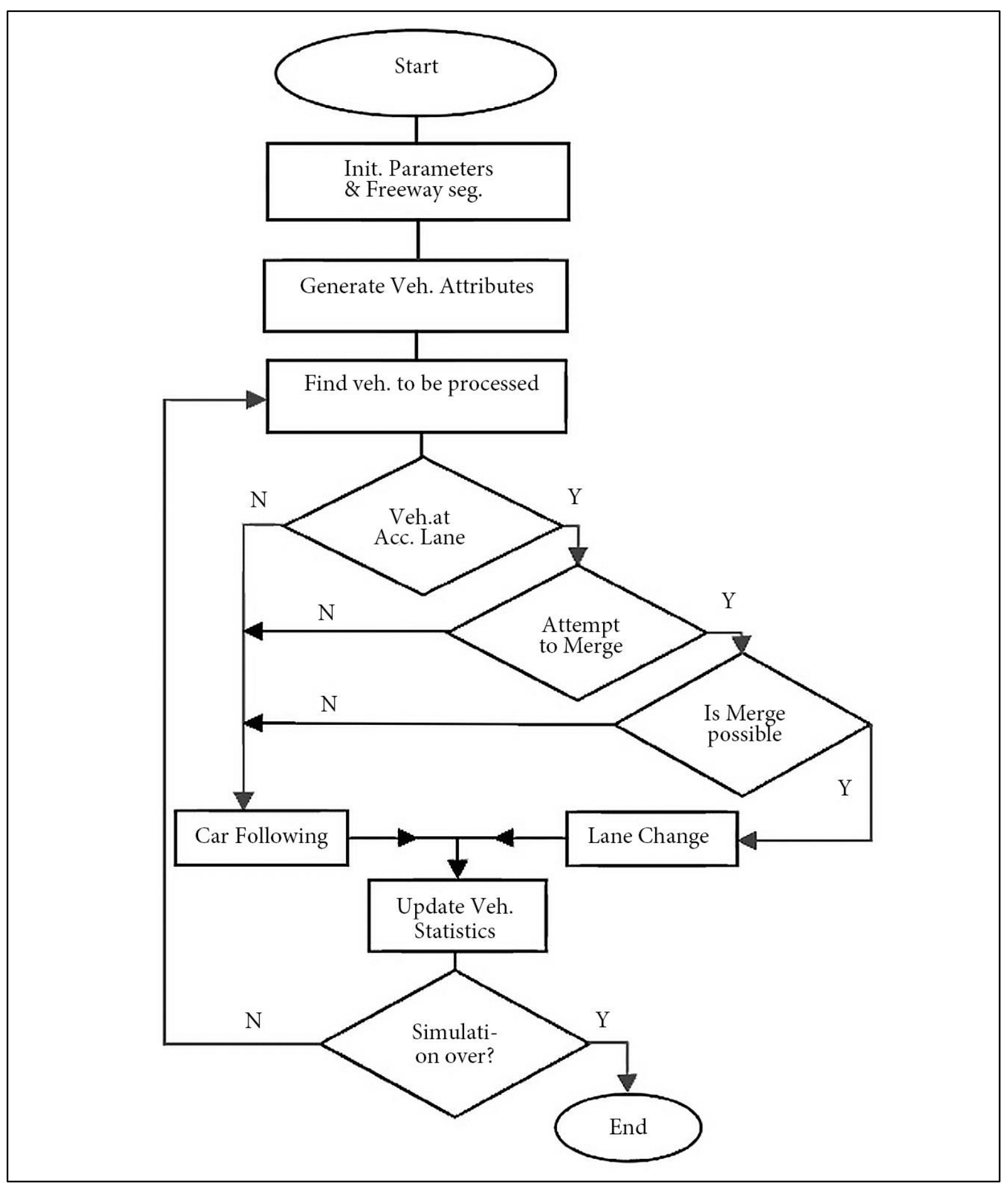

Figure 1. The logic of on-ramp vehicle simulation 


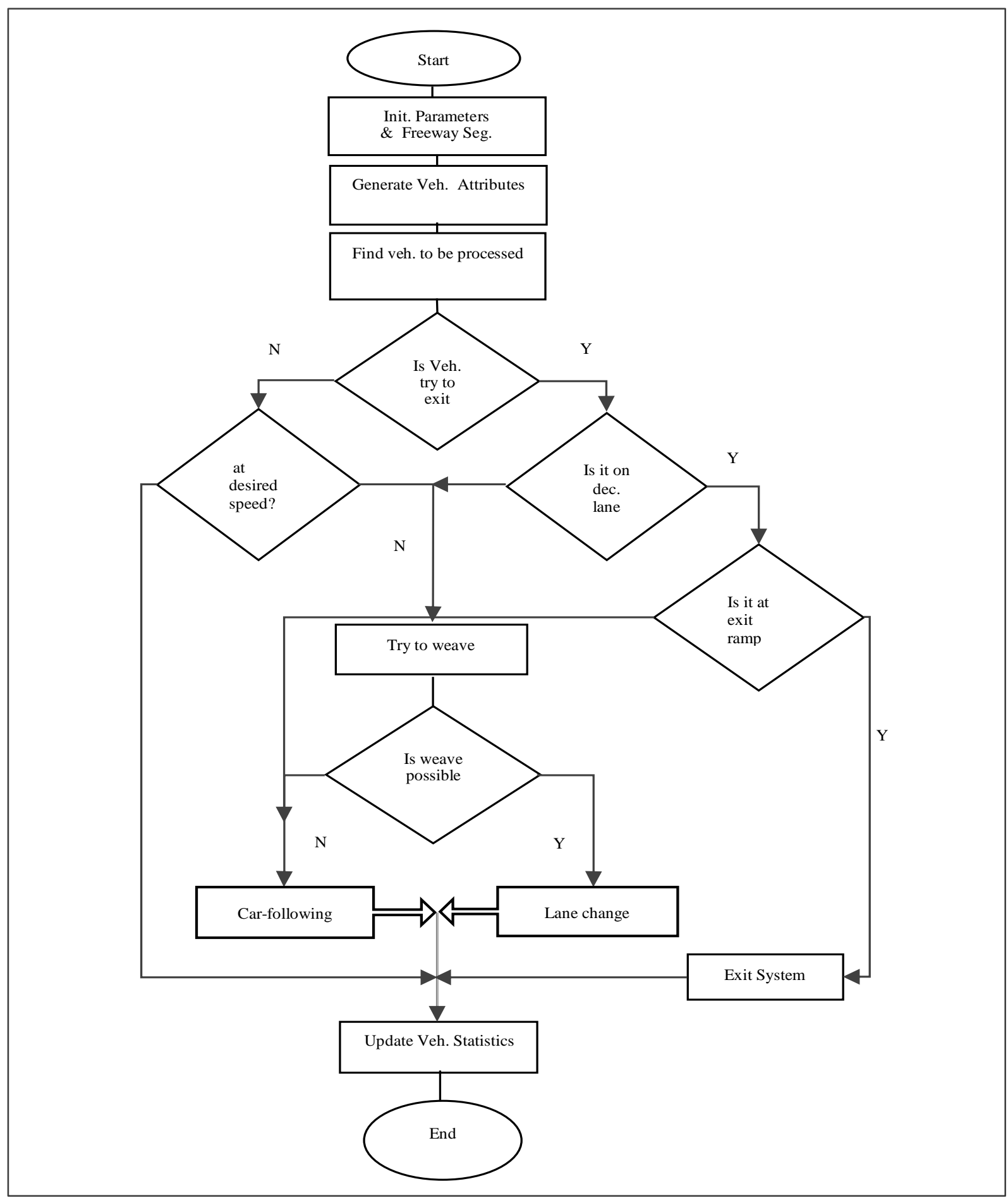

Figure 2. The logic used for freeway vehicle simulation

\section{Car-following Logic}

The car-following process is important to simulate the traffic flow, analyze the formation mechanism of traffic congestion, and manage the alternative proposals [13]. The car-following process takes place when "a driver follows a lead vehicle and tries to maintain distance and relative speed within an acceptable range".

Sultan et al. conducted a research to investigate whether drivers are able to use information on the acceleration or deceleration of the lead vehicle during the car-following process. The authors collected field data for car-following process using an instrumented vehicle. A strong evidence was concluded that the drivers are able to realize the lead vehicle's acceleration or deceleration [11].

The car-following process in FWASIM was considered when vehicles are at a distance of $(60 \mathrm{~m})$ or less. This value was obtained from the observed results. The maximum 
distance considered for car following logic is the product of the average observed preferred headway and the maximum observed speed increased by $10 \%$. The increment is attributed to the fact that with the video recording technique, it is hard to get an accurate data representing the real behavior due to the fixed installation of the camera that will not cover all driver behavior diversity.

ILLISIM proposed a distance of $76 \mathrm{~m}$ or less between the lead and follow vehicles for car following logic [5]. Herman and potts observed that driver's behavior is not affected when the spacing between vehicles is greater than $61 \mathrm{~m}$ in car-following [4].

In car-following process, drivers try to adjust their speed to keep a separation equal to their desired spacing. Desired spacing is the product of the speed of the follower vehicle and preferred headway. Preferred headway is the time a driver has, during steady state car following and represents the driver's ability to develop the same amount of deceleration as the lead vehicle in case the leader breaks [6].

The main parameters that affect driver behavior in car following process are the desired spacing and the relative speeds of vehicles. Vehicles accelerate, decelerate or maintain speed depending on their desired spacing and relative speeds.

Three cases of leader and follower vehicles are considered at the simulation model:

1. When the speed of the follower vehicles is less than their desired speed, then they accelerate to reach their desired speed;

2. When spacing is less than the desired, vehicles decelerate if the leader speed is equal to or less than the follower speed. For the same case, vehicles coast when the speed of the leader is greater than the follower speed; and
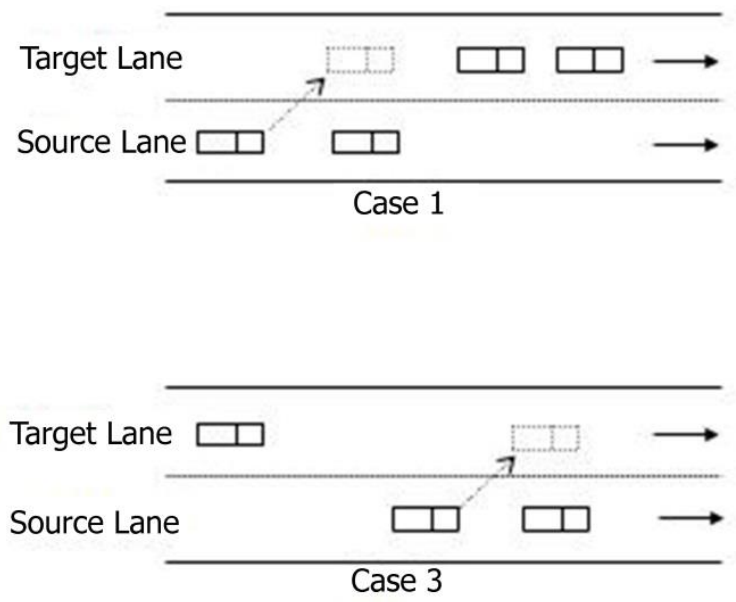

3. When the spacing is greater than the desired spacing of the follower vehicle and its speed is less than its desired speed, in this case vehicles adopt acceleration maneuver.

\section{Lane Changes Maneuver}

Lane changes are classified as either mandatory Lane Change (MLC), for example, a required lane change, or discretionary Lane Change (DLC) as in case of a driver trying to improve his driving conditions. Lane change process was simulated according to the following:

Multilane highways (up to six lanes in this simulation model) are scanned and processed in each time step (one second) from left to the right and then from the right to the left for every two adjacent lanes. Lane change process is randomly occurred for each case depending on simulated traffic conditions.

The successive case was then checked if it fits any of the four main cases that frequently occur and simulated to represent the real site situations. The four cases were categorized based on the traffic availability in the target lane. The four main cases are graphically illustrated in Figure (3).

Lane changing maneuver under both MLC and DLC types was applied in the current simulation model as a sequence of four steps:

1. Driver decision to apply a lane change, in case of the subject vehicle speed is less than its desired speed and it is a weaving vehicle type,

2. Choice of a target lane, this depend on the available gaps and also depend on the vehicle weaving type,

3. Acceptance of lead and lag gaps, and

4. Performing the lane change maneuver.
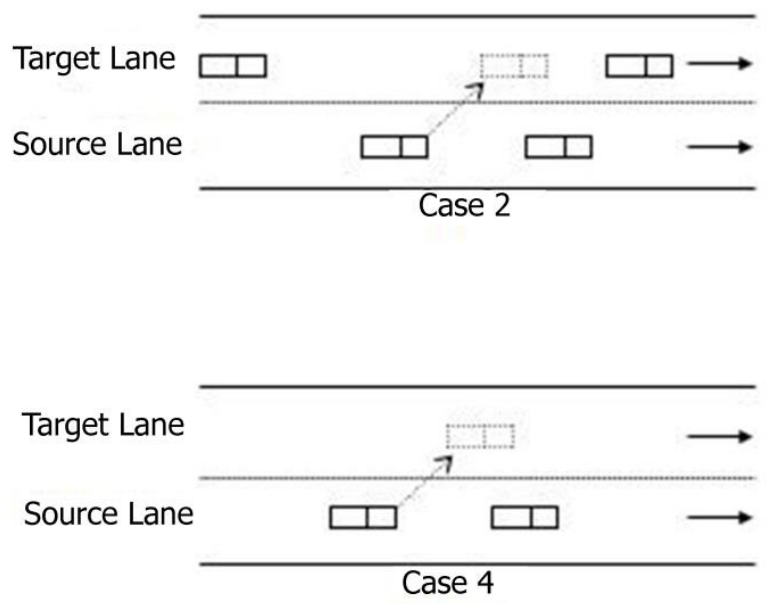

Figure 3. The four main lane change cases 


\section{Modeling Segment Geometry}

\subsection{Modeling On-ramp Geometry}

The on-ramp in this model is considered consisting of one lane. This lane is assumed sufficient to allow for the passage of only one row of vehicles in one direction. The on-ramp lane was assumed to meet the highway link by at start of the acceleration lane. The meeting point represents the on-ramp vehicle generation. It is considered as the origin of the simulated vehicle on the On-ramp. The weaving type determines the destination of each vehicle.

\subsection{Modeling Link Geometry}

The modeled highway link or the main road of freeway consists of up to six lanes in the simulation model indicated by numbers starting from one for the far side lane. This number will remain as a label for each vehicle entering the simulated area. To achieve flexibility in modeling a range of possible freeway layouts, the number of lanes is specified to the simulation model through input.

The vehicle generation for these lanes starts from the beginning of the link at a point just before reaching the on-ramp nose by a given distance specified to the simulation model by input.

The segment length computation starts from the vehicle generation point (origin point) which includes the followings:
1. The nose length, which is the distance between the origin point and the meeting point of on-ramp with the highway link;

2. The acceleration lane length (auxiliary lane), is used by the on-ramp vehicle to merge.

3. The weaving area length, which is the distance from the nose to the off-ramp position, by which the acceleration and the deceleration lane lengths are involved.

4. The sign position, is an implicitly distance within the weaving area length needed for the exit sign to be installed a distance before the off-ramp (exit position); and

5. The deceleration lane length that is the lane used by the exit vehicles before leaving the freeway through the off-ramp.

The above lengths were specified to the simulation model by input. The other geometric parameters such as gradient and curvature were not considered at this stage of the model development. Figure (4) represents typical cross section showing the highway geometric parameters.

\subsection{Modeling Off-ramp Geometry}

The off-ramp geometry is assumed to consist of one lane. This configuration allows for movement of raw of vehicles in the highway exit direction. During the simulation model input stage, the user should specify a number equal to (1) to represent the number of off-ramp lanes. The off-ramp lane is assumed to meet the highway link at the end of the deceleration lane.

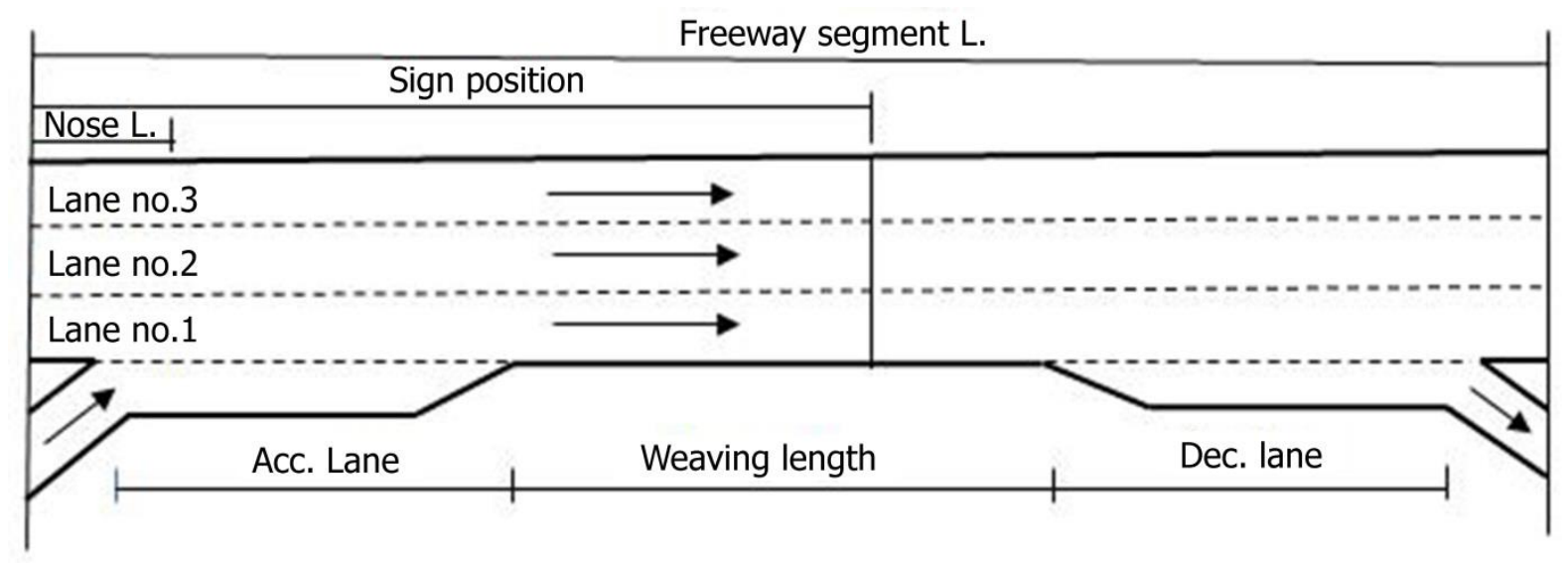

Figure 4. Typical proposed freeway segment cross section 


\subsection{Modeling Traffic Data}

Microscopic models permit measurement of a vast range of traffic features and allow many traffic management strategies to be tested. Because the developed model is microscopic type, therefore, the majority of the important and effective vehicle attributes and driver characteristics are modeled and specified to the model by input.

In general, traffic characteristics may be classified into three main categories;

1. Static characteristics include physical and fixed vehicle attributes, such as size and type.

2. Dynamic characteristics, which represent the degree of alert of a driver and his response as he may face an incident. and

3. Kinematic characteristics represent the vehicle performance and driver characteristics, such as acceleration and deceleration rate.

The traffic data which were modeled in the current simulation model represent collection of the above three categories.

An important assumption made in this study is that the different behavioral characteristics, such as desired speed, acceleration rate, critical lags and gaps, etc. of a vehicle-driver are generated independently. In the real world situation, it is more probable that a driver with a high desired speed will accelerate and decelerate faster, and has a smaller critical lag and gap than a driver with a lower desired speed.

Vehicle attributes and driver features which were modeled are described hereafter [3];

- Type and arrival time of the generated vehicle.

- The vehicle movement type, if it is weaving or non-weaving vehicle.

- Vehicle acceleration rate. and desired speed.

- Driver preferred headway, brake reaction time, and accepted gap.

- $\quad$ Spacing and desired spacing.

- Vehicle label.

- Entry lane number

\subsection{Vehicle Arrivals}

Vehicle arrivals are provided by means of vehicle generators considered at a distance on freeway segment before on ramp nose. This distance is specified to the model by input. Numbers are generating from uniform distribution of an interval $(0,1)$. The model using the transformation given below calculates times between successive vehicle arrivals;

1. In case of negative exponential distribution

$$
\underline{\log (R n d)}
$$

2. In case of shifted negative exponential distribution

$$
\log (\text { Rnd })[(-)
$$

Where,

$t$ is the generated time interval in seconds,

Rnd is a random number distributed uniformly in the range $(0,1)$, and

$q$ is the vehicle flow in veh/sec.

\subsection{Vehicle Desired Speed}

The desired speed of a driver is the speed on which he/she try to maintain. The following important variables are affecting the desired speed [1]:

- Highway geometry.

- The quality of pavement surface.

- Weather conditions.

- The limited speed of the specific highway section.

- Density of traffic in front of the vehicle.

- Vehicles speed in front of the simulated vehicle.

- Traffic composition.

- type of the vehicle, and

- The driver characteristics.

Each simulated vehicle assigns desired speed generated from a normal distribution. The values of the normal distribution, which are; mean, standard deviation (STD), minimum, and maximum values are specified during the input stage. In specifying the upper and lower limit of the desired speed, bounds are set to the distribution curve. The technique of lane specific values for the desired speed is considered.

It is also assumed that drivers moving on the far side lane have low range of variation that is low standard of deviation. By contrast, it was assumed a high range of variation of desired speed for drivers moving on the near side lane.

\subsection{Type of Vehicle Movement (Weaving and Non-weaving Vehicles)}

According to their movement type, the generated vehicles are either weaving or non-weaving type. Based on the comparison with a random number generated from the uniform distribution, the vehicle is considered either weaving or non-weaving.

To achieve the logic in the developed simulation model, the proportions of weaving and non-weaving vehicles should be provided during the data input stage. One of the outputs of this followed logic is the determination of vehicle destinations. This is because, vehicles originating from highway link and considered as weaving vehicles then their destination must be the off-ramp exit. Similarly, weaving vehicles originating from on-ramp will continue their movement with the non-weaving vehicles originated from the highway link. This method is applicable for both vehicles generated from on-ramp and those from highway link. 


\subsection{Traffic Composition}

The traffic composition has a substantial effect on the operating characteristics. Values of traffic composition for highway flow and on-ramp flow are held in a cumulative form. In the simulation model, whenever vehicle is generated, a random fraction of unity is generated according to a uniform distribution. Generated random number is divided according to the cumulative form into parts. Each part represents vehicle type proportion. If vehicle type proportions are not supplied, the program applies default values given in Table (1). The default values were concluded from the average observed values during the study period conducted on different highway sites at Baghdad city.

Table 1. Default vehicle type proportions

\begin{tabular}{|c|c|c|c|c|}
\hline Vehicle type & Passenger cars & Buses & Trucks & Others \\
\hline Proportion & 0.90 & 0.03 & 0.06 & 0.01 \\
\hline
\end{tabular}

\subsection{Acceleration and Deceleration Rate}

The acceleration rate depends mainly on vehicle capability and performance. The simulation model adopted Gipps acceleration model. Equation (3) below explains Gipps model [8].

$$
V_{m}=V+2.5 * a c c * T *\left(1-\frac{V}{V_{d}}\right) *\left(0.025+\frac{V}{V_{d}}\right)^{\frac{1}{2}}
$$

Where,

$V_{m}$ is the maximum speed to which vehicle can accelerate,

acc is the generated acceleration rate,

$T$ is the time step in seconds,

$V$ is the speed of the subject vehicle, and

$V_{d}$ is the desired speed of the subject vehicle.

Normal acceleration rate is drawn from a normal distribution generated randomly for each simulated vehicle. The observed values for acceleration and deceleration rates are shown in Table (2).

Table 2. Parameters of normal acceleration rate

\begin{tabular}{|c|c|c|c|c|}
\hline Rate & $\begin{array}{c}\text { Mean } \\
\mathrm{m} / \mathrm{s}^{2}\end{array}$ & $\mathrm{STD}$ & $\begin{array}{c}\mathrm{Min}^{\mathrm{m}} \\
\mathrm{m} / \mathrm{s}^{2}\end{array}$ & $\mathrm{Max}^{\mathrm{m}} \mathrm{m} / \mathrm{s}^{2}$ \\
\hline Acceleration & 2.69 & 1.70 & 0.7 & 5.2 \\
\hline Deceleration & 2.66 & 1.45 & 0.85 & 5.0 \\
\hline
\end{tabular}

\subsection{Spacing Gap}

When vehicle is generated, each driver assigns a critical gap drawn from a normal distribution. In order to compute the available spacing used in the car following logic, the gap value is transformed into distance (meters) and supplied during the input stage.

\subsection{Time Preferred Headway Modeling}

When a vehicle is generated, each driver assigns a preferred headway drawn from a normal distribution. The value of preferred headway is used to calculate the desired spacing used for car following logic adopted in the developed simulation model.

\subsection{Modeling of Break Reaction Time}

Brake reaction times can be expected from drivers who have to brake suddenly and unexpectedly. The sudden brake results in a time delay in a driver's response, or in other means, it is the response lag. It includes both the perception and reaction times. Identification of these two times separately at the observed data is difficult, therefore the break reaction time term represents the summation of the two. When a vehicle is generated during the model simulation process, each driver is assigned a value of break reaction time drawn from a normal distribution.

\section{Simulation Model Verification and Validation}

The simulation model verification and validation test process can be split into three stage [2]:

1. Software testing often called model verification,

2. Assessment of overall performance of the model validation, and

3. Hypothesis testing and modification, called model calibration.

\subsection{Model Verification}

The process of model verification is to determine if the logic of model listing as described by the model developer is compatible with the computer code, and to check if the input data produces the desired and accurate results (output data) in terms of magnitude and direction [10].

The verification process for the current simulation model is made in two sequential steps. The first step involves compiling the program, running it for error-free and with only the necessary mathematical approximation. The current computer model was written with visual c++language. This language technique has debugging facility, which allows the execution of the program to be controlled so that the developer can monitor specific locations, variables, change of array dimensions etc., and check the sequence of program control. After tracking down the mistakes, the source program was edited to produce a new version. The procedure was repeated as long as an error is detected and corrected.

After establishing the internal correctness of the model, a number of tests were carried out to establish a measure of confidence in the model performance, which represents the second step of the verification process. Selection of the 
values of input parameters is involved through the test step. These values enclose the expected domain of application of this model.

Verification process can be performed depending on data rather than of field data. However, considering of field data will ensure that the verification is achieved for some input parameters, which are similar with field conditions.

\subsection{Simulation Model Validation}

Analytical validation and field validation processes were conducted to test the validity of FWASIM. These two processes are fully explained in the following sections;

\subsubsection{Analytical validation}

A simple hypothetical freeway weaving section was conducted to validate FWASIM. Tests were based on comparison of FWASIM output with the output of VISSIM. The selection of test was made to consider the important factors, which may affect the vehicle behavior for a given freeway, segment configuration. The HCS2000 software is used to calculate the capacity of the weaving segment.

Proposed weaving sections were specified to be examined under different vehicle parameters and various flow conditions with different weaving area configurations. The following is an example for one of the proposed sections explaining how the simulation model was analytically validated. The objective of this test is to examine the model performance by simulating the behavior of traffic stream through a weaving section and then compare the results with that resulted when the same proposed weaving section is simulated by VISSIM.

The geometric layout of the proposed weaving section and the percentage of the weaving flow are shown in the Figures (5) and (6) respectively. The weaving segment capacity was $4380 \mathrm{veh} / \mathrm{h}$ as computed by HCS2000 program for the same prevailing conditions.

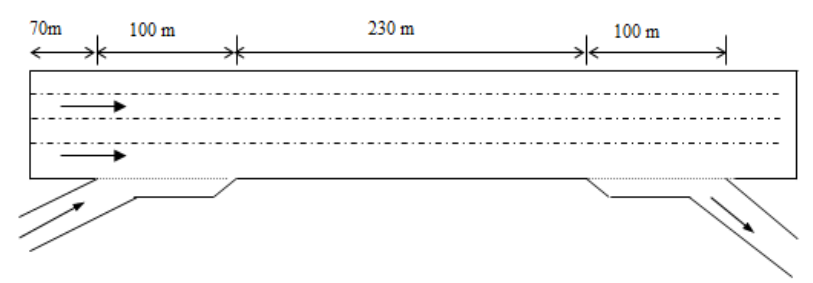

Figure 5. The geometric layout of the weaving section

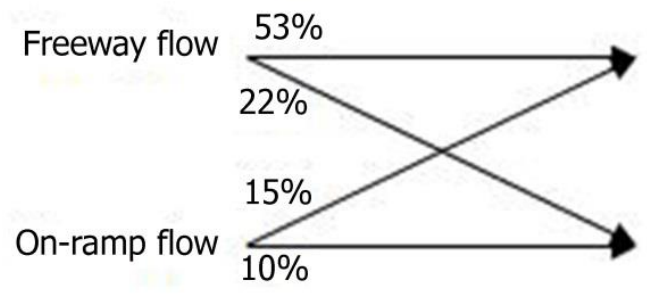

Figure 6. the flow-weaving diagram

The average desired speed of vehicles in the weaving section was chosen to be the measure of effectiveness parameter to compare the results. Figures (7) shows the VISSIM schematic of test site.

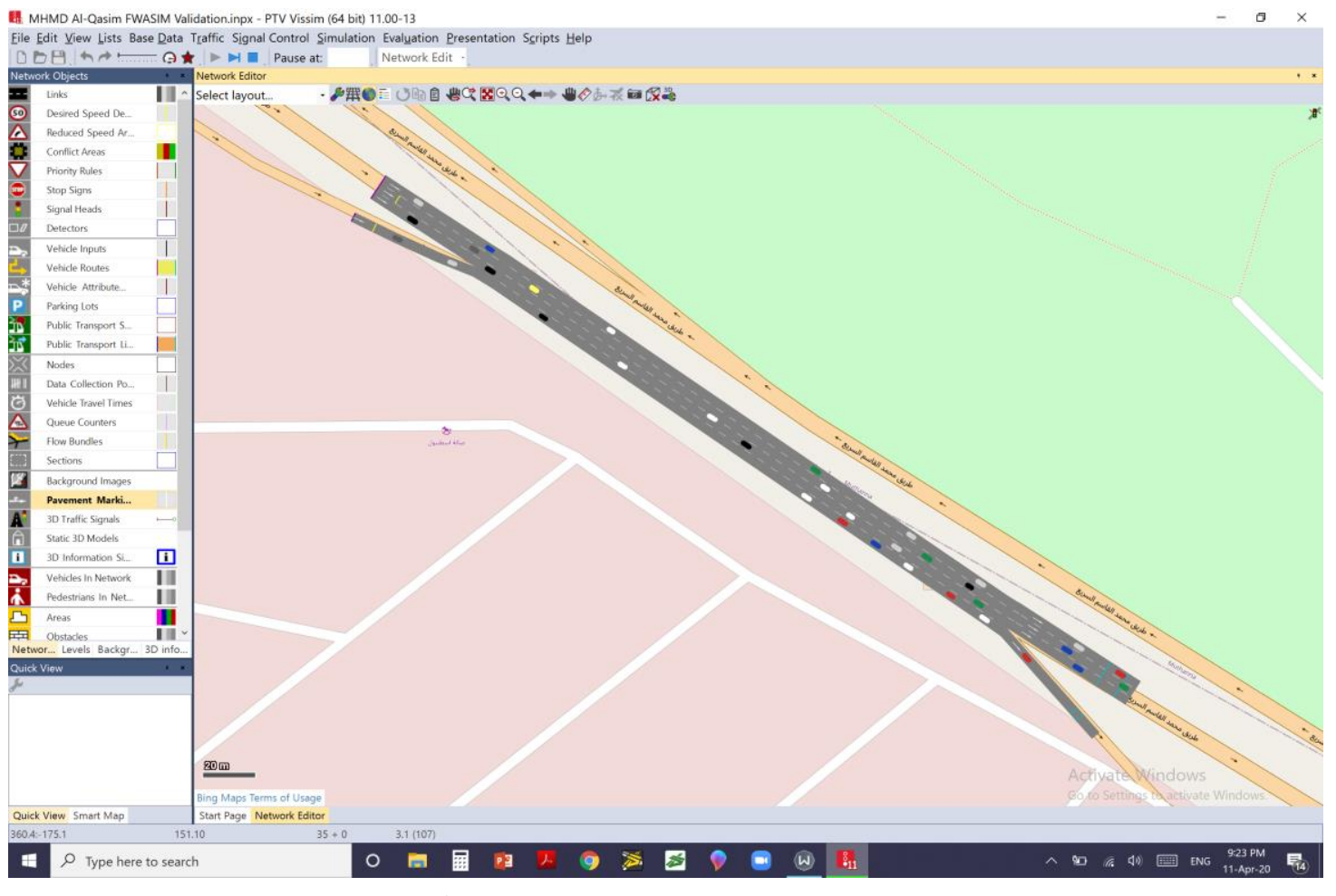

Figure 7. VISSIM schematic of proposed site 
The desired speed FWASIM simulation results are compared with that resulted by VISSIM. Figures (8) illustrates and compares the results with the corresponding traffic volume starting from $500 \mathrm{veh} / \mathrm{h}$ to the determined section's capacity.

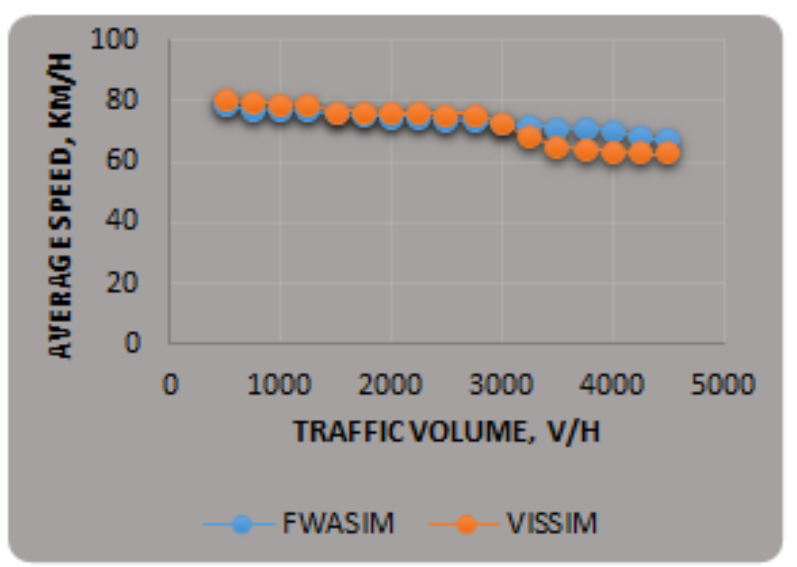

Figure 8. Average desired speed comparison results

Figure (8) indicates a tendency for the simulation model to overestimate traffic speed near the section's capacity. This simulation model behavior is attributed to the FWASIM input method of driver behavior that represents the gap, brake reaction time, and the preferred headway. These attributes are not assigned during the VISSIM input stage. Figure (9) explains the FWASIM input for driver and vehicle attributes.

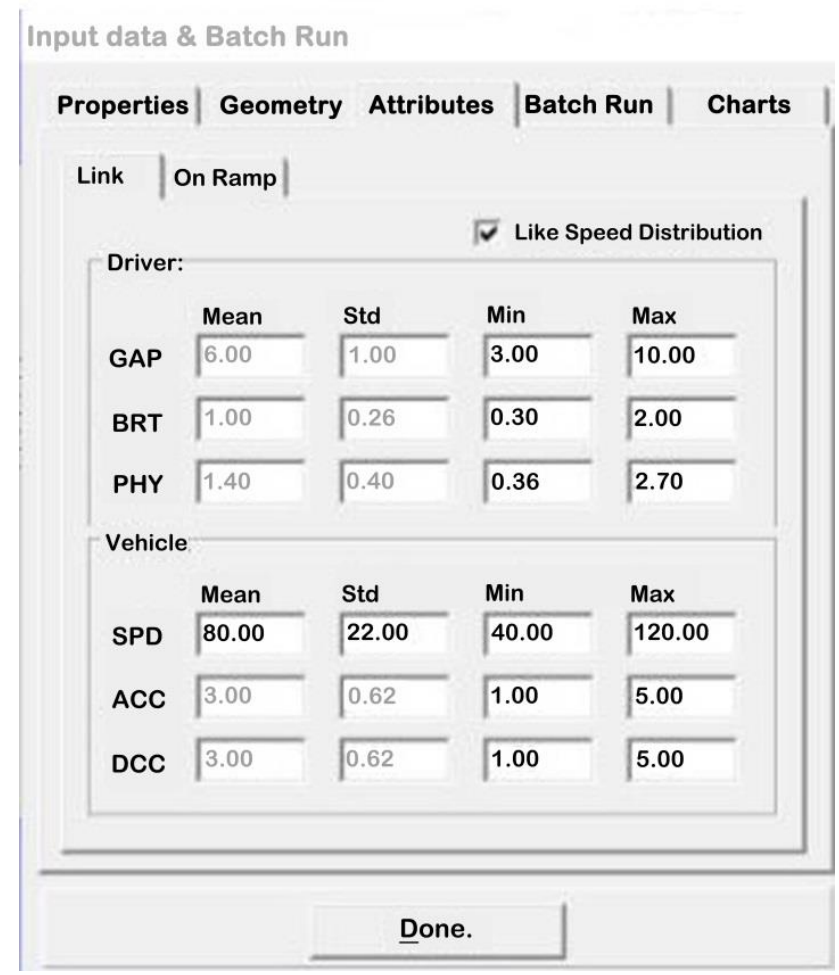

Figure 9. FWASIM traffic attribute input
Because of the simulation modeling of FWASIM was built based on the observed field data, then the difference in outputs of traffic parameter values will be attributed to the great varieties in driver behavior, vehicle characteristics. Driver decision plays an important role in the position and the speed of each vehicle, which is also influenced by external factors relating to the roadway geometry and traffic interaction. Therefore, the conclusion for the above section is that the simulation model is may considered analytically validated.

\subsubsection{Simulation model field validation}

Model validation considers, as "the process of determining to what extent the model's underlying fundamental rules and relationships are able to adequately capture the targeted behavior, as specified within the relevant theory and as demonstrated by field data". In other words, can the driver's behavior or traffic feature rules used by the model produce the relative traffic performance such as capacities, densities, and the effect of weaving [10].

Consequently, it is necessary to compare the simulated results from the model with the observed data from survey sites. Validation is the process checking the overall model-predicted traffic performance for a facility against the observed field data of traffic performance not used in the calibration stage [7].

Travel time (TT) was selected as a sensitive traffic parameter to be used for simulation model validation purpose. TT is defined as "the time difference between the time of vehicle leaving the weaving area and its initial time by which vehicle entering that area". Measurement of TT may implicitly reflect vehicle delay through highway section. For the observed TT calculation, data were collected from different sites other than that used in the simulation model development stages. The observed vehicle TT was classified into four categories according to origin-destination concept. Each category represents one type of vehicle movement. Table (3) shows these categories and their corresponding vehicle movement type.

Table 3. Different categories with their vehicle movement type.

\begin{tabular}{|c|c|c|}
\hline \multirow{2}{*}{ TT category } & \multicolumn{2}{|c|}{ Movement type } \\
\cline { 2 - 3 } & Origin & destination \\
\hline 1 & Link & Link \\
\hline 2 & Link & Off-ramp \\
\hline 3 & On-ramp & Link \\
\hline 4 & On-ramp & Off-ramp \\
\hline
\end{tabular}

Computer programs were used as an aid tools to collect and abstract data from videotape. Their outputs were processed and analyzed to compute average vehicle TT for the different categories. The observed traffic parameter values were specified to the simulation model as an input. Table (4) shows these observed parameters with their statistical values. The observed passenger car percentage 
was $90 \%$.

Table 4. Statistical values for observed input parameters

\begin{tabular}{|c|c|c|c|c|}
\hline Parameter & Mean & STD & $\operatorname{Min}^{\mathrm{m}}$ & $\mathrm{Max}^{\mathrm{m}}$ \\
\hline Speed, $\mathrm{km} / \mathrm{h}$ & 75 & 23 & 40 & 135 \\
\hline Acc. rate, $\mathrm{m} / \mathrm{s}^{2}$ & 2.70 & 1.70 & 0.75 & 5.2 \\
\hline Dec. rate, $\mathrm{m} / \mathrm{s}^{2}$ & 2.60 & 1.45 & 0.85 & 5.0 \\
\hline $\begin{array}{c}\text { Preferred headway, } \\
\text { sec. }\end{array}$ & 1.40 & 0.40 & 1.0 & 2.1 \\
\hline $\begin{array}{c}\text { Break reaction time, } \\
\text { sec. }\end{array}$ & 1.17 & 0.26 & 0.60 & 2.10 \\
\hline
\end{tabular}

The simulation model was run several times with the observed values for each session of the collected data to obtain the simulated average TT for each category (origin-destination type). The simulated vehicular TT and the observed results for all sessions are presented in Table (5).

The comparison of the simulated and observed results is graphically illustrated in Figure (10). The line $\mathrm{x}=\mathrm{y}$ has been superimposed in the plot to facilitate comparison. The mean for observed and simulated TT are not matching exactly. However, they are close enough to establish a level of confidence that the model is capable of simulating a freeway weaving area operation.

Table 5. Observed and simulated values of TT

\begin{tabular}{|c|c|c|c|c|c|c|c|}
\hline \multicolumn{4}{|c|}{ Observed TT, sec. } & \multicolumn{4}{|c|}{ Simulated TT, sec. } \\
\hline cat.1 & cat.2 & cat.3 & cat. 4 & cat.1 & cat.2 & cat.3 & cat. 4 \\
\hline 14.0 & 14.5 & 15.0 & 14.5 & 14.5 & 15.0 & 14.8 & 15.0 \\
\hline 13.5 & 13.0 & 14.0 & 13.5 & 14.0 & 13.5 & 14.2 & 14.0 \\
\hline 12.5 & 12.5 & 13.5 & 13.0 & 12.0 & 17.0 & 13.8 & 13.5 \\
\hline 15.5 & 15.0 & 18.6 & 16.4 & 16.0 & 15.8 & 16.2 & 16.5 \\
\hline 16.7 & 17.0 & 19.5 & 18.4 & 15.5 & 17.5 & 17.6 & 17.5 \\
\hline 16.0 & 15.5 & 18.0 & 17.6 & 15.2 & 15.4 & 17.5 & 17.0 \\
\hline 17.5 & 16.5 & 14.5 & 15.0 & 16.0 & 17.5 & 15.5 & 15.5 \\
\hline 17.0 & 18.5 & 16.0 & 15.5 & 16.5 & 16.3 & 16.5 & 15.5 \\
\hline 18.0 & 18.0 & 17.5 & 16.5 & 19.0 & 18.5 & 16.8 & 17.0 \\
\hline 19.0 & 18.5 & 15.5 & 16.0 & 18.2 & 18.4 & 17.2 & 17.0 \\
\hline 19.0 & 19.5 & 16.5 & 17.0 & 18.0 & 18.5 & 16.0 & 18.0 \\
\hline 17.5 & 17.0 & 15.5 & 15.0 & 16.5 & 16.2 & 16.5 & 16.0 \\
\hline 18.6 & 18.5 & 17.5 & 17.0 & 18.0 & 17.6 & 16.5 & 16.5 \\
\hline 19.5 & 19.0 & 18.0 & 18.0 & 19.0 & 18.5 & 17.5 & 17.5 \\
\hline 20.0 & 20.5 & 19.5 & 19.0 & 19.0 & 21.0 & 18.7 & 18.8 \\
\hline 21.0 & 19.5 & 18.0 & 17.5 & 20.0 & 20.5 & 18.5 & 18.0 \\
\hline 22.5 & 21.5 & 19.5 & 18.5 & 22.0 & 22.0 & 20.4 & 18.8 \\
\hline 25.0 & 24.5 & 24.5 & 23.5 & 24.0 & 24.2 & 23.8 & 24.2 \\
\hline 28.5 & 27.0 & 26.5 & 26.0 & 18.8 & 28.0 & 26.5 & 25.5 \\
\hline 30.5 & 31.0 & 28.5 & 29.0 & 29.2 & 30.5 & 30.0 & 28.8 \\
\hline 32.0 & 31.5 & 30.0 & 29.0 & 31.8 & 31.0 & 31.0 & 29.5 \\
\hline 35.0 & 34.0 & 33.5 & 32.5 & 34.5 & 34.6 & 33.0 & 33.0 \\
\hline 36.0 & 34.5 & 35.0 & 34.5 & 35.7 & 35.5 & 34.5 & 33.8 \\
\hline 36.5 & 37.5 & 38.0 & 33.0 & 38.0 & 39.5 & 36.5 & 39.5 \\
\hline
\end{tabular}



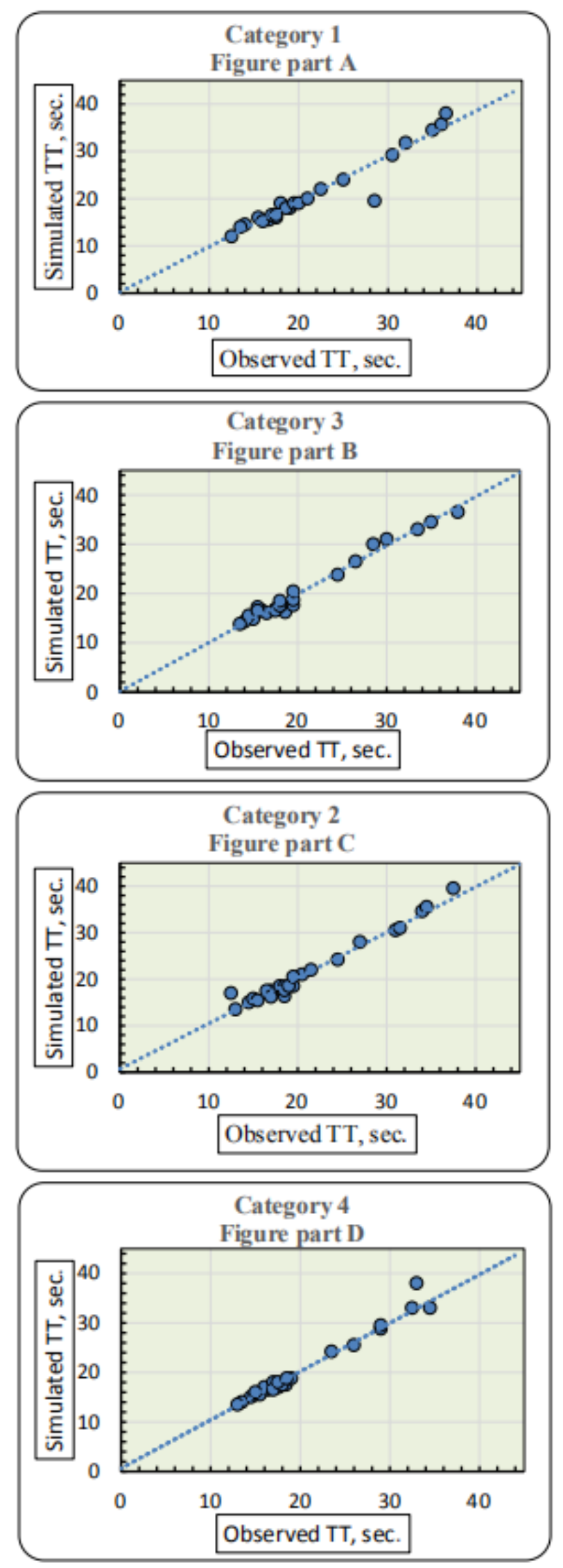

Figure 10. Part A to D Comparison between observed and simulated TT for all movement type
T-test was used to validate the results. The observed and simulated average mean values were calculated and found to be (21.08) and (21.04) seconds respectively. At the 5\% level of significance and 23 degrees of freedom, the calculated $t$ value is $(0.027)$.

The corresponding tabulated $t$ value, that is, the critical $t$ is (1.71) which is higher than the calculated t. This has the implication that there is no reason at $95 \%$ level of significance, to reject the hypothesis that the observed results may be represented by the simulated results. This implies that the results are statistically significant and the model provides reasonably accurate measures of effectiveness for the validated range of input data.

\section{Results}

Analytical validation is conducted by specifying proposed weaving sections to be examined by FWASIM and VISSIM under various traffic features, flow conditions, and different weaving area configurations. Their results of comparison showed an acceptable agreement. However, results indicated a tendency for the simulation model to overestimate traffic speed near the section's capacity. This behavior is attributed to the FWASIM input method of driver behavior that represents the gap, brake reaction time, and the preferred headway, which are not assigned during the VISSIM input stage.

Field validation produced that the mean for observed and simulated TT are not matching exactly. However, they are close enough to establish a level of confidence that FWASIM is capable of simulating a freeway weaving area operation.

\section{Conclusions}

This study focused on developing microsimulation program to measure the performance of the weaving sections at the freeway, where the traffic conflict occur due to the weaving process. The followings can be concluded;

1. Microscopic traffic simulation model is the suitable tool to develop vast numbers of online traffic management strategies instead of field testing solutions, which are costly and cumbersome.

2. FWASIM output for predicting traffic stream models (speed-flow-density relationship) at weaving sections was agreed with that predicted by VISSIM program. However, there was a tendency for the simulation model to overestimate the traffic speed near the section capacity. The reason was attributed to the difference in driver decisions and traffic charactrisitics adopted by the two softwares.

3. FWASIM could be applied to analyze and design highway sections with a range of geometric configurations under different local traffic conditions. 


\section{Recommendation}

It is recommended to calibrate FWASIM for more sensetive input traffic parameters, in case of applying FWASIM in an area other than the area of study, which is the Republic of Iraq, Baghdad city.

\section{REFERENCES}

[1] Ahmed, Kazi Iftekhar. Modeling Drivers' Acceleration and Lane Changing Behavior. Ph.D, Massachusetts Institute of Technology, USA, 1999.

[2] Ali, Al Neami, and Alkubaisi Mahdi. Study of Vehicle Behavior at Signal Controlled Junctions. College of Engineerin, University of Baghdad Journal of Engineering,Vol. 6 No. 4, 2000.

[3] Alkubaisi, Mehdi. Simulation Modeling of Traffic Behavior at Speed Change Lanes. Ph.D, University of Baghdad 2004.

[4] Aycin, M, and R Benekohal. Linear Acceleration Car-Following Model Development and Validation. Transportation Research Record: Journal of the Transportation Research Board, no. 1644: 10-19, 1998.

[5] Bham, Ghulam H, and Rahim F Benekohal. Illisim, a fast high fidelity traffic simulation model based on cellular automata and car-following concepts. Transportation Research Board 80th Annual Meeting 2001.

[6] Bham, Ghulam H Benekohal, Rahim F. A High Fidelity Traffic Simulation Model Based on Cellular Automata and
Car-Following Concepts. Transportation Research Part C: Emerging Technologies 12 (1): 1-32, 2004.

[7] Division, VDOT traffic Transportation. VDOT Vissim User Guide Version 2.0, no. January 2020.

[8] Jimenez, T, P Mussi, and G Siegel. A Road Traffic Simulator: Car-Following and Lane-Changing. European Simulation Multiconference May 2000 (Lc): 241-45, 2000.

[9] Koppula, Nanditha, and Antonio A Trani. A Comparative Analysis of Weaving Areas in HCM , A Comparative Analysis of the Weaving Areas in HCM, TRANSIMS, CORSIM, VISSIM and INTEGRATION. Integration The Vlsi Journal, no. April 2002.

[10] Rakha, Hesham, Bruce Hellinga, Michel Van Aerde, and William Perez. Systematic Verification, Validation and Calibration of Traffic Simulation Models. In 75th Annual Meeting of the Transportation Research Board, Washington, DC. Citeseer 1996.

[11] Sultan, Beshr, Mark Brackstone, and Mike McDonald. Drivers' Use of Deceleration and Acceleration Information in Car-Following Process. Transportation Research Record: Journal of the Transportation Research Board, no. 1883: 3139,2004

[12] S. Kim, W. Suh and J. Kim, Traffic Simulation Software: Traffic Flow Characteristics in CORSIM. International Conference on Information Science \& Applications (ICISA), Seoul, 2014, pp. 1-3, doi: 10.1109/ICISA.2014.6847475.

[13] Zhang, Yong, Ping Ni, Minwei Li, Hao Liu, and Baocai Yin. A New Car-Following Model Considering Driving Characteristics and Preceding Vehicle's Acceleration. Journal of Advanced Transportation. https://doi.org/10.115 5/2017/2437539, 2017 